\title{
Diseño de laboratorio lógico programable
}

\section{Programmable logic lab design}

\author{
GONZÁLEZ-MONZON, Ana Lilia†*, PACHECO-ALVARADO, Luis Kevin y AGUILAR LOPEZ, \\ Alfredo
}

Tecnológico de Estudios Superiores de Jilotepec, México.

ID 1 ${ }^{\text {er }}$ Autor: Ana Lilia, González-Monzón / ORC ID: 0000-0002-0280-0525, CVU CONACYT ID: 151293

ID $1^{\text {er }}$ Coautor: Luis Kevin, Pacheco-Alvarado / ORC ID: 0000-0001-5845-4150, CVU CONACYT ID: 883154

ID $2^{\text {do }}$ Coautor: Alfredo, Aguilar-López / ORC ID: 0000-0002-7279-9048, CVU CONACYT ID: 1090739

DOI: $10.35429 /$ JOCT.2020.13.4.24.29

Recibido: Abril 25, 2020; Aceptado: Junio 30, 2020

\section{Resumen}

En el presente trabajo las tecnologías emergentes requieren un entrenamiento rápido en las mismas para procesamiento de SOC (System On Chip), con lo que han mejorado características como consumo de potencia, diseño de sistemas y velocidad de procesamiento, por lo cual se requieren elementos electrónicos que ayuden con el tiempo de aprendizaje y practica de dichos temas por lo tanto el objetivo es diseñar un prototipo de laboratorio lógico programable en el Tecnológico de Estudios Superiores de Jilotepec considerando la naturaleza del proyecto, se desarrolló mediante una metodología que guiará paso a paso las actividades como la CDIO que se utiliza en el área de las ingenierías la cual consta de cuatro etapas: concepción, diseño, implementación, la contribución principal es coadyuvar a los alumnos a realizar sus prácticas por medio de una placa circuito impreso o PCB que básicamente es un soporte físico en donde se encuentran todos los componentes electrónicos y digitales que se utilizan en las diferentes materias como electrónica digital, programación básica, programación avanzada, control digital, electrónica analógica, las cuales requieren utilizar los dispositivos para conocer su funcionamiento de interconexión y por medio de la programación al realizar las prácticas que lo conforman utilizando el menos tiempo posible para realizar cada una.

Lógico, Programable, Electrónica

\begin{abstract}
In the present work, emerging technologies require rapid training in them for SOC (System On Chip) processing, which has improved characteristics such as power consumption, system design and processing speed, which is why electronic elements that help with the learning and practice time of these topics, therefore the objective is to design a prototype of a programmable logic laboratory at the Tecnológico de Estudios Superiores de Jilotepec considering the nature of the project, it was developed through a methodology that will guide step to I pass the activities such as the CDIO that is used in the engineering area which consists of four stages: conception, design, implementation, the main contribution is to help students to carry out their practices through a printed circuit board or PCB that basically it is a physical support where all the electronic and digital components that are They are used in different subjects such as digital electronics, basic programming, advanced programming, digital control, analog electronics, which require using the devices to know their interconnection operation and through programming when carrying out the practices that make it up using the least possible time to carry out each one.
\end{abstract}

Citación: GONZÁLEZ-MONZON, Ana Lilia, PACHECO-ALVARADO, Luis Kevin y AGUILAR LOPEZ, Alfredo. Diseño de laboratorio lógico programable. Revista de Tecnologías Computacionales. 2020. 4-13:24-29.

\footnotetext{
* Correspondencia del Autor (Email: mtra.analilia@tesji.com.edu)

$\uparrow$ Investigador contribuido como primer autor.
} 


\section{Introducción}

Con la realización de un diseño de laboratorio lógico programable, el cual está constituido por medio de una placa circuito impreso o PCB que básicamente es un soporte físico en donde se instalan componentes electrónicos, eléctricos y digitales en donde se interconectan por medio de pistas, constituida de los componentes como Matriz de led 8x8, display de 7 segmentos, relevador de potencia, buzzer, display LCD, $\mathrm{WiFi}$, Bluetooth, sensor humedad y temperatura, sensor ultrasónico, potenciómetro, motor DC, puente $\mathrm{H}$, joystick, sensor PIR, resistencias, botones y leds también incluye una tabla protoboard, los cuales por su funcionalidad pertenecen a diferentes familias de circuitos. Este prototipo con esas características no se encuentra en el mercado, y es considerando una propuesta útil en las materias de electrónica digital, programación básica, programación avanzada, control digital, electrónica analógica, por lo tanto, los estudiantes podrán realizar sus prácticas de manera eficiente y más rápida insertando codificación correcta para cada uno de los componentes, esto lograra facilitar la tarea de la conexión de dispositivos al realizar cada práctica. En primer apartado se encuentra los antecedentes de la concepción, en el segundo los elementos principales del laboratorio lógico programable y las familias de los dispositivos, en el tercero los pasos a seguir o etapas utilizadas mediante la metodología CDIO, en el cuarto apartado se encuentra el diseño electrónico, en el cuarto la implementación del tipo de programación de FPGA con el lenguaje de programación VHDL, en el quinto operación contiene resultados del diseño de laboratorio lógico programable por ultimo comentarios y referencias

\section{Antecedentes}

En el Tecnológico de Estudios Superiores de Jilotepec en la carrera de Ingeniería Mecatrónica dentro de la retícula los alumnos toman materias que requieren tecnologías que se encuentran a la vanguardia como electrónica digital, programación básica, programación avanzada, control digital, electrónica analógica, entre otras las cuales con llevan el realizar prácticas muy extensas con poco tiempo para realizarlas generalmente utilizan dispositivos electrónicos como microcontroladores, display, servo motores, sensores entre otros, los costos son altos y no siempre el alumno cuenta con el recurso económico para su adquisición.

\section{Elementos del laboratorio lógico programable}

\section{Sistemas electrónicos}

Los sistemas electrónicos es un conjunto de circuitos que interactúan entre sí cuyo objetivo es controlar el funcionamiento de dispositivos o maquinas con ayuda de transistores, diodos, circuitos integrados, entre otros, donde los sensores o transductores son dispositivos electrónicos que toman una magnitud física y los convierten en una señal eléctrica para su posterior procesamiento, mediante una tarjeta de desarrollo y poder manipular dispositivos como actuadores y poder visualizar los resultados en un dispositivo de salida como un display, un LCD o un sistema de luces mediante LEDs.

\section{Circuitos electrónicos}

Son aquellos componentes como resistencias, condensadores, fuentes entre otros los cuales son conectados eléctricamente entre sí para alimentar los dispositivos procesando las señales de entrada y salida. La corriente eléctrica es un movimiento de electrones, por lo tanto, cualquier circuito debe permitir el paso de los electrones por los elementos que lo componen con una trayectoria cerrada que permita fluir continuamente como un FPGA, pantalla Lcd 16X2, display de 8 segmentos 4 dígitos Ánodo común, Bluetooth, WiFi, diodos led, Matriz 8X8 con driver Max 7219, buzzer. Malvino (2017).

\section{Transductores o sensores}

Son considerados es un dispositivo capaz de transformar o convertir una determinada magnitud física con una señal eléctrica que pueda ser manipulada arrojando una diferente de salida, pero con valores muy pequeños en términos relativos con respecto de un generador como son un sensor de humedad DHT22, sensor ultrasónico HC-SR04, sensor PIR y un puente H TB6612FNG. 


\section{Plataforma electrónica}

FPGA (Dispositivo programable que contiene bloques de lógica) de muy bajo coste de la familia Cyclone II de Altera, esto otorga la ventaja de que puede realizarse un procesamiento a muy alta velocidad estos dispositivos se utilizan en los llamados "lenguajes de descripción de hardware", sus interconexiones, para crear diferentes funciones lógicas, ya sean combinacionales o secuenciales. Es un dispositivo programable en campo o FPGA contiene bloques de lógica con una conexión y funcionalidad que puede ser configurada mediante lenguaje especializado de hardware y programación, donde se usa desde una compuerta lógica hasta sistemas combinacionales complejos, tiene la ventaja de ser reprogramables ya que cuenta con funciones de alto nivel basadas RAM volátiles en ROM reprogramables son basadas en EPROM o flash. $\mathrm{y}$ las no programables basadas en fusible o antifusibles. (Ver figura 1).

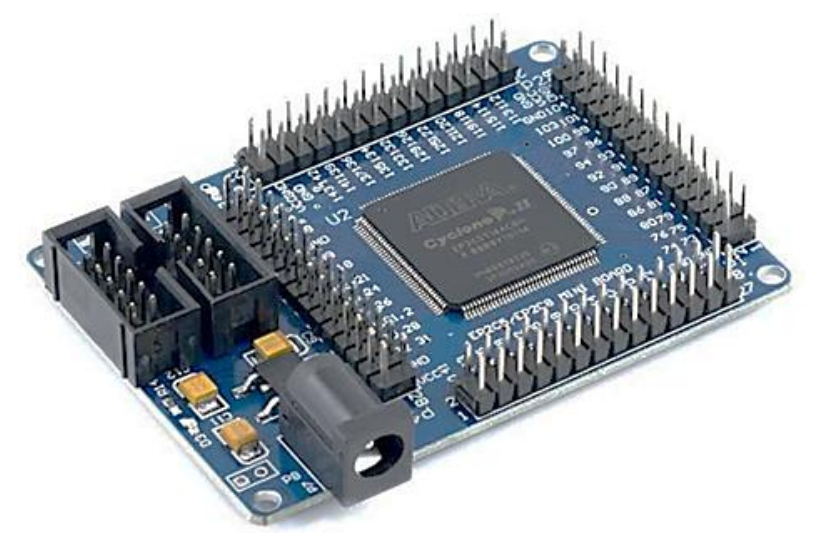

Figura 1 FPGA

- Un FPGA contiene múltiples bloques lógicos $22 \mathrm{~V} 10$.

- Componentes: Logic Block; Programmable Interconnect; I/O.

- $\quad$ Interconexión por matriz.

- Interconexión con multiplexor por cada entrada del bloque lógico.

\section{Elementos Mecánicos}

Son elementos accionados de forma manual, como contactos que al juntarse permiten el paso de la corriente eléctrica, como los que se mencionan a continuación.

\section{- Botón}

Es un interruptor de presión, en el cual hay de dos tipos normalmente abierto o normalmente cerrado, en donde en su accionar puede ser que permita el paso de la corriente eléctrica o no dependiendo de su configuración.

\section{- Interruptor}

Es un dispositivo mecánico donde una posición es la que marca que está apagado y en la posición contraria esta encendido. Este tipo de interruptor es más usado para sistema de protección en donde al ponerlo en posición de apagado, desconecta de la corriente eléctrica cualquier dispositivo o aparato que esté conectado con él en serie, o lo mantiene encendido, pero también se puede usar como un dispositivo de lógica, en donde el interruptor al estar en apagado es un 0 (cero) o al estar en encendido es un 1 (uno).

\section{- $\quad$ Potenciómetro}

Es un tipo de resistencia que es variable, el cual cuenta con tres terminales de las cuales dos son el valor de la resistencia en fijo, y una es la parte variable, ya sea que se conecte con el extremo de una de las dos terminales de donde se tendrá una fracción de su diferencia de resistencia. (ver Figura. 2).

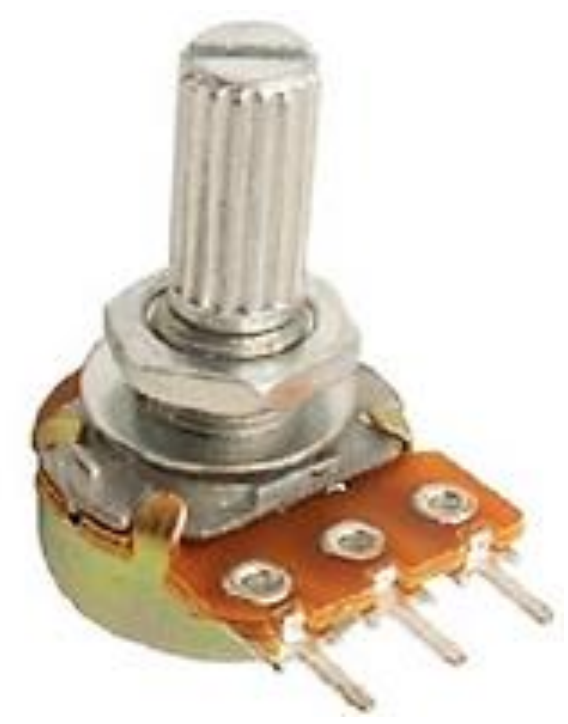

Figura 2 Potenciómetro 
- $\quad$ Palanca (joystick)

La palanca (joystick) es un dispositivo que en su arreglo incorpora dos potenciómetros que, en su movimiento horizontal y vertical, un potenciómetro para cada movimiento, cambia el valor resistivo de los potenciómetros, con lo cual se puede permitir la variación de potencial. Este dispositivo es muy útil, porque permite el control de dispositivos normalmente electromecánicos como motores, los cuales son usados para el movimiento de forma vehicular o control de velocidad.

\section{Elementos electromecánicos}

La electromecánica es la combinación de las ciencias del Electromagnetismo de la ingeniería eléctrica y la ciencia de la mecánica. (Camacho 2003)

\section{- $\quad$ Relevador}

Este dispositivo es un elemento que incorpora en su construcción un elemento eléctrico y un elemento mecánico que, al ser excitado eléctricamente, genera un movimiento mecánico en cual mueve con una lámina de cobre que sirve de contacto que sube o baja mediante la excitación eléctrica y este movimiento mueve un contactor, que permiten la conexión y desconexión de la conducción eléctrica.

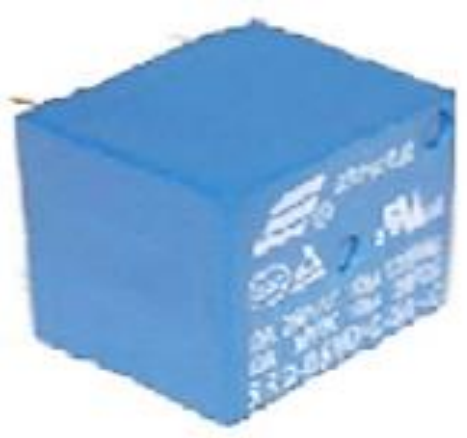

Figura 3 Relevador

\section{- $\quad$ Motor CD}

Es un dispositivo eléctrico que desarrolla una fuerza contra electromotriz, que consta de dos piezas, el estator que es la armadura externa del motor y el rotor, lo que hace este dispositivo es convertir una magnitud eléctrica en un movimiento mecánico rotatorio, dependiendo de su uso en el diseño de cualquier estructura que requiera movimiento.

\section{- $\quad$ Motor PaP}

Este motor es un dispositivo electromecánico que convierte una serie de pulsos electrónicos en desplazamientos angulares que varían desde los $90^{\circ}$ hasta pequeños movimientos de 1.8" lo que significa que es capaz de girar en pasos un ángulo determinado solamente, a diferencia de los motores de corriente continua que no pueden quedar en una sola posición, mientras los motores pasan a paso sí pueden guardar la posición.

\section{- Servomotor}

Estos son dispositivos que contiene en su interior un encoder, conocido como decodificador que convierte el movimiento mecánico en pulsos digitales interpretados por un controlador de movimiento llamado driver que ambos forman un circuito para dar velocidad y posición.

\section{Metodología}

En el desarrollo se aplicó la metodología CDIO para prototipos y proyectos de ingeniería complementados con procesos industriales $\mathrm{y}$ determinar el tiempo idóneo del proyecto. Consta de cuatro fases:

- Concepción, la etapa para recabar la información necesaria de la estructura, tamaño, grosor de las pistas, pero también determinar los componentes necesarios el así como el software de diseño a utilizar y las consideraciones técnicas pertinentes.

- Diseño, determina, tamaño, material y conexiones para analizar la trasferencia de datos y ubicación de dispositivos en el diagrama.

Implementar la programación de la placa con FPGA para cada uno de los dispositivos por medio de VHDL para ver la conexión y validación de las terminales.

- Operar utilizar la programación para funcionamiento de los dispositivos con elementos secundarios. 
Esta fue diseñada específicamente para ser adaptado en actividades auténticas a través de conocimientos que permite alcanzar los objetivos. Frente a los nuevos retos de productividad. (Ver figura 4).

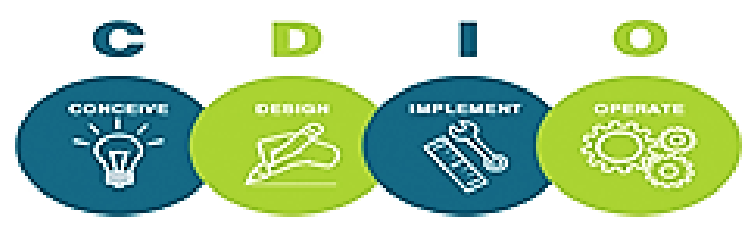

Figura 4 CDIO

\section{Diseño electrónico}

El tamaño de la placa es 33.4 largo x 20.1de ancho En esta se puede ver cada uno de los dispositivos conforman el laboratorio y las respectivas conexiones que las cuales entran a un proceso de análisis para verificar las conexiones de los dispositivos de manera correcta y no puedan provocar daños de ningún tipo en los dispositivos ni en las conexiones de cada uno de ellos (ver Figura 5).

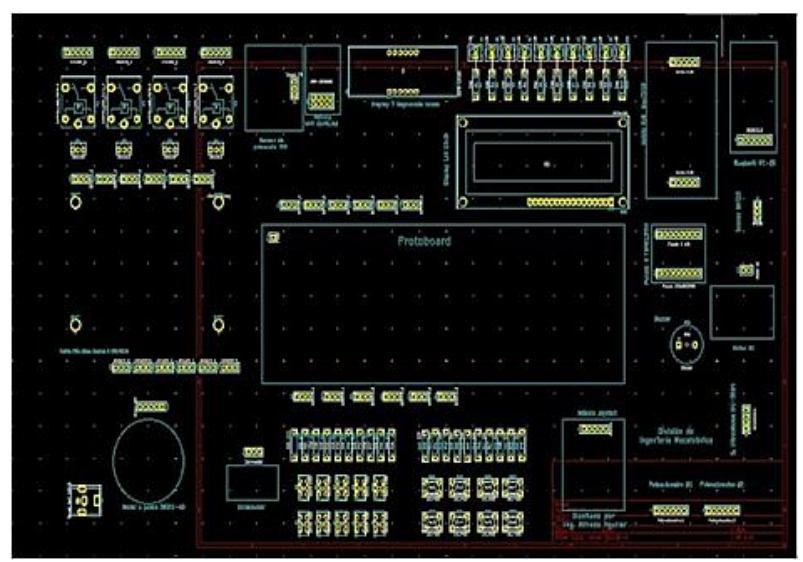

Figura 5 Diseño electrónico

Fuente: Elaboración propia

\section{Implementación de programación}

El lenguaje de descripción de Hardware VHDL (Very High Speed Integrated Circuits) sirve para optimizar diferentes tipos desarrollos de dispositivos lógicos programables, la cual se rige en una arquitectura de 5 pasos que son Biblioteca, Entidad, Arquitectura, Paquetes y Componentes (Maxines, 2014). Pero también posee una estructura de programación amigable y fácil de entender, es por esto que el lenguaje se utiliza para la programación del FPGA la funcionalidad de los dispositivos por ejemplo (Ver tabla 1).

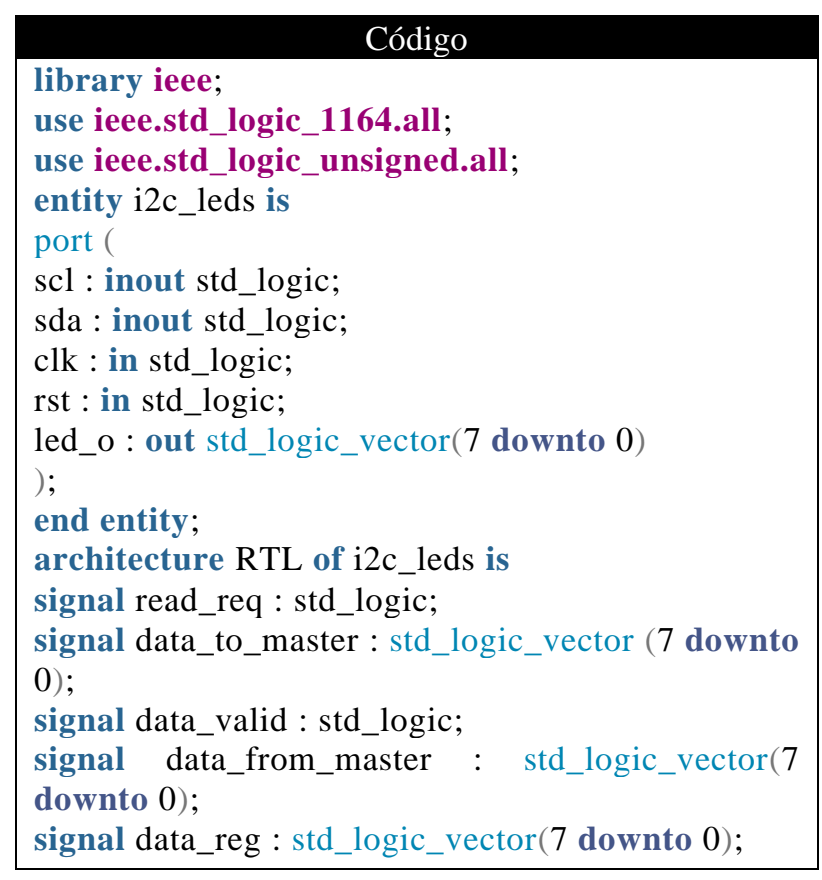

Tabla 1 Código FPGA con VHDL

\section{Resultados}

En el resultado del diseño del Laboratorio Lógico Programable, constituido por una placa PCB y 21 dispositivos en los que se encuentra sistemas electrónicos, circuitos electrónicos, transductores y plataforma electrónica, elementos mecánicos que se alimenta de 5 vlt por medio de un Jack, está conformada por dos capas para evitar corto circuitos y asegurar su funcionamiento óptimo en las conexiones integrando una protoboard para ampliar las funciones con más dispositivos que puedan existir en el mercado y no se encuentren integrados, como tarjeta de desarrollo un FPGA de esta manera cuenta con los elementos principales que se utilizan para realizar prácticas de cada una de las materias como son: electrónica digital, programación básica, programación avanzada, control digital, electrónica analógica. Los dispositivos se encuentra insertados en una PCB, la cual está conformada por dos capas, para darle libertad a las pistas para un correcto funcionamiento electrónico, evitando cortos circuitos su interconexión se realiza por medio de una tabla protoboard para hacer las conexiones pertinentes con el FPGA hacia cada uno de los dispositivos, un display de 7 segmentos, display de 16 × 2, un joystick, motor a pasos modelo MOT-130, botones, módulo Bluetooth, sensor temperatura y humedad, sensor ultrasónico, potenciómetro, joystick, sensor PIR, resistencias, matriz, modulo Wifi, módulo bluethoot, entre otros (ver Figura 6). 


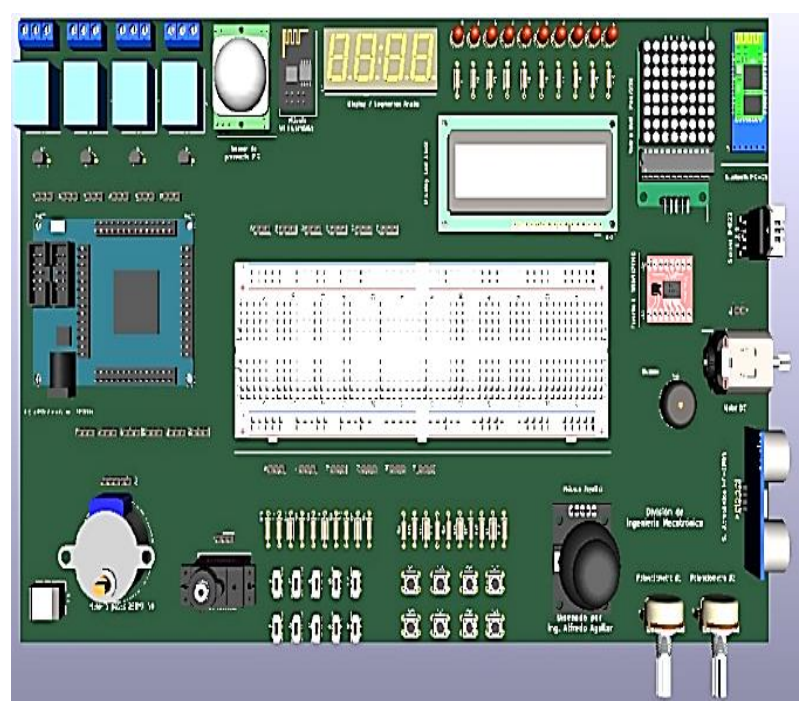

Figura 6 Prototipo

La figura muestra como está constituido el Laboratorio Lógico Programable, a la ubicación de los dispositivos y la interconexión entre cada uno estos.

\section{Conclusiones}

La importancia de este prototipo coadyuvan a la integración de los 21 diferentes dispositivos integrados en una PCB los cuales son los más utilizados como base para realizar las prácticas de diferentes temarios de cada una de las asignaturas que involucran la electrónica, como utilidad principal del prototipo es apoyar a los estudiantes a realizar en el menor tiempo posible las practicas al tener en el Laboratorio Lógico Programable todos los elementos necesarios pero también un aprendizaje significativo al optimizar la forma de realizar las practica. obteniendo tiempo para otras actividades y proyectos.

\section{Agradecimientos}

Agradecemos al Tecnológico de Estudios Superiores de Jilotepec por el patrocinio, apoyo y facilidades otorgadas para participar en este congreso.

También al departamento de Mecatrónica por todo el apoyo otorgado para realizar el diseño de este prototipo.

\section{Referencias}

Garza.G.J. A (2017) Sistemas digitales y electrónica digital prácticas de laboratorio, México, Pearson educación Prentice hall
Gaviria Arbeláez, C. (2017). Arepas colombianas: técnicas profesionales de cocina. Universidad de La Sabana Colombia.

Hermosa D.A. (2018), Principios de electricidad y electrónica I, México D.F. Alfa omega.

Morales E.F., Sucar L.E. (2019) Los robots del futuro y su importancia para México, Ciudad de México.

https://ccc.inaoep.mx/ emorales/Papers/2019/e duardo.pdf

Maxines G. D. (2014) Programación de sistemas digitales con VHDL, Ciudad de México, grupo editorial patria S.A de C.V.

Malvino A. Bates J. D. (2017) principios de electrónica, Madrid España, McGraw-Hill. 